\title{
How Should Complementary Practitioners and Physicians Communicate? A Cross-Sectional Study from Israel
}

\author{
Eran Ben-Arye, MD, Moshe Scharf, MD, and Moshe Frenkel, MD
}

Objectives: The extensive use of complementary and alternative medicine for patients can complicate dialogue between physicians and complementary and alternative medicine practitioners, but not much data have been collected on the expectations and attitudes of physicians and complementary and alternative medicine practitioners concerning their communication and collaboration. In this study, we compared the results of a cross-sectional survey of both groups to elucidate the attitudes and expectations regarding communication and collaboration.

Methods: Questionnaires were mailed electronically or through the mail to 2532 primary care physicians and 450 complementary and alternative medicine practitioners employed by Clalit Health Services, the largest health maintenance organization in Israel.

Results: Questionnaires were returned by 333 physicians (response rate of 13\%) and 241 practitioners (response rate of 54\%). According to our results, the majority of both groups expressed an interest in clinical practice collaboration $(69 \%$ and $77 \%$ of physicians and practitioners, respectively; $P=$ $.043)$; preferred using a medical letter to communicate with each other; and expected to consult with each other about mutual patients to formulate treatment plans. However, the practitioners were more interested than the physicians in collaborative scientific research $(15 \%$ vs $42 \%$, respectively; $P<.0001)$ and collaborative medical education ( $2 \%$ vs $27 \%$, respectively; $P<.0001)$. The physicians also supported a physician-guided model of teamwork in clinical practice, whereas the practitioners supported a more collaborative model.

Conclusions: Educational programs for primary care physicians and complementary and alternative medicine practitioners should focus on aspects of communication between the groups and practical methods for writing referral or medical letters. (J Am Board Fam Med 2007;20:565-571.)

Complementary and alternative medicine (CAM) encompasses various therapeutic methods and techniques, some of which have their origins in traditional and philosophical systems of medicine.

This article was externally peer reviewed.

Submitted 3 May 2007; revised 29 June 2007; accepted 3 July 2007.

From The Complementary and Traditional Medicine Unit, Department of Family Medicine, Faculty of Medicine, Technion-Israel Institute of Technology, Haifa, Clalit Health Services, Haifa and Western Galilee District (EB-A, MF); Clalit Mashlima - Complementary Clalit Health Services, Israel (MS); and the Integrative Medicine Program, The University of Texas, M. D. Anderson Cancer Center, Houston (MF).

Funding: none.

Conflict of interest: none declared.

Corresponding author: Eran Ben-Arye, MD, The Complementary and Traditional Medicine Unit, Department of Family Medicine, Clalit Health Services, 6 Hashahaf Street, Haifa 35013, Israel (E-mail: eranben@netvision.net.il).
Some scholars have named any merging of CAM with conventional biomedicine "integrative medicine," whereas others perceive CAM as "a higherorder system, or systems, of care that emphasizes wellness and healing of the entire person as primary goals, drawing on both conventional and CAM approaches in the context of a supportive and effective physician-patient relationship."

The increasingly widespread use of CAM is throwing into greater relief problems with communication among CAM practitioners, physicians, and their patients. ${ }^{2,3}$ Advocates of integrative medicine have acquired evidence to support the benefits of collaboration between mainstream medicine and CAM in clinical practice, scientific research, and medical education. ${ }^{4,5}$ Several studies have shown a gap in communication between physicians and patients that was associated with low disclosure rates 
of patient use of CAM treatments and the reluctance of physicians to make referrals for CAM treatments. ${ }^{6,7}$ Two studies that have yielded such evidence examined physicians' and acupuncturists' reports and found CAM referral rates to be lower than $30 \%$ in the United States. ${ }^{8,9}$ In another US study, $82 \%$ of 517 primary care physicians reported they had strong referral relationships with other primary care physicians but lacked direct, formalized referral relationships with chiropractors. ${ }^{10} \mathrm{~A}$ survey of 2875 members and fellows of the United Kingdom's Royal College of Physicians found that $41 \%$ referred patients to CAM. ${ }^{11}$ However, the fact that $32 \%$ of the respondents practiced CAM themselves and that the United Kingdom's National Health Service (NHS) includes several CAM modalities may explain why the United Kingdom's CAM referral rate is higher than that of the United States.

Research-based integrative medicine was a key component of the United States's National Center of Complementary and Alternative Medicine's 2001 to 2005 strategic plan. ${ }^{12}$ In addition, an increasing number of collaborative studies are being designed and published by physicians and nonphysician CAM researchers who are often affiliated with CAM research centers within medical facilities. ${ }^{13}$ Some progress is being made on several fronts. For example, in the United Kingdom, the Prince of Wales advocated the integration of various CAM modalities into the NHS. ${ }^{14}$ The recent Smallwood report, which examined the role of CAM in the NHS, recommended that a full-assessment of CAM therapies and their potential role within the NHS be performed and suggested the provision of health care on an integrated basis. ${ }^{15}$ Collaborations between physicians and non-physician medical educators are also increasing, as evidenced by the fact that courses on CAM-related topics were offered during the 2002 to 2003 academic year at some of the 98 medical schools in the United States. ${ }^{16}$

The more widespread emergence of integrative medicine initiatives in medical practice, research, and education raises a number of important questions regarding the communication between physicians and CAM practitioners: Do physicians and CAM practitioners share a common interest in clinical, scientific, or educational collaboration? Are they willing to communicate with each other about the patients they have in common? What are their expectations regarding this communication and possible collaborative teamwork? How do they envision CAM being integrated in medical practices, such as primary care clinics? To answer these questions, we compared the results of a cross-sectional survey of both groups.

\section{Research Methods \\ Study Sites and Participants}

We performed a 2-arm study of primary care physicians and CAM practitioners employed by Clalit Health Services (CHS), the largest of 4 health maintenance organizations in Israel. CHS serves 3,800,000 clients, which constitutes approximately $60 \%$ of the Israeli population. ${ }^{17}$ All 4 of Israel's health maintenance organizations offer CAM treatments under medical surveillance through administratively separate agencies, which were created because of economic considerations. In 2005, the 4 health maintenance organizations' CAM agencies conducted $45 \%$ of the 1.4 million CAM treatments performed in Israel. ${ }^{18}$

Of the 4 CAM agencies, the one operated by CHS is the largest. It offers patient-paid, reducedprice CAM services at 40 clinics throughout Israel. Although the CAM agencies' services are generally not covered by the state medical insurance, CHS partially reimburses patients for the costs of CAM treatments, but only if patients have supplementary medical insurance. Most of the clinics offer a variety of CAM treatments, including herbal and nutritional supplements; systematic systems of complementary medicine (eg, traditional Chinese medicine and homeopathy); and manual healing. Although some of its clinics are located in conventional primary and secondary care clinics, they operate independently, with their own administrative and clinical structures. In most cases, patients visit the CAM agencies without referral letters from their physicians. In general, no mechanism exists for the health maintenance organizations' CAM practitioners and conventional physicians to communicate or establish referral patterns and other professional peer relationships.

\section{Study Design}

The study was constructed based on research experience and discussions with physicians, CAM students and practitioners, and patients in both conventional and CAM clinics in Israel and Texas. The 
study consisted of 4 stages: (1) questionnaire construction, (2) primary questionnaire refinement, (3) secondary questionnaire refinement, and (4) survey administration.

The questionnaire was constructed based on a comprehensive literature review and discussions at meetings of the Complementary and Traditional Medicine Unit's administrative staff.

To make the questionnaire as comprehensible as possible we conducted 2 focus group discussions: one with 14 physicians (consisting of residents and specialists practicing family medicine in urban and rural primary care clinics) and one with 15 CAM practitioners practicing a wide range of CAM modalities, including naturopathy, herbal medicine, acupuncture, and manual therapies. In the first of 2 discussion components, the participants were asked to suggest the main areas of interaction between physicians and CAM practitioners and to formulate potential questions and answers for the questionnaire. In the second component, the participants were asked to react to previously formulated topics and questions.

The secondary refinement of the questionnaire was based on the focus groups' appraisals. Because it was more likely to be understood, the authors used a broad definition of CAM: "therapies often named alternative, complementary, natural, folk/ traditional medicine, which are not usually offered as part of the medical treatment in the clinic." Added to this definition was a list of CAM modalities, which included herbal medicine; Chinese medicine (including acupuncture); homeopathy; folk and traditional medicine; diet/nutritional therapy (including nutritional supplements); chiropractic; movement/manual healing therapies (eg, massage, reflexology, yoga, Alexander and Feldenkreis techniques); mind-body techniques (eg, meditation, guided imagery, relaxation); energy and healing therapies; and naturopathy.

Survey administration consisted of mailing or e-mailing questionnaires to the 2532 primary care physicians and 450 CAM practitioners employed by CHS. Survey data were entered into a computer database for further analysis.

\section{Data Analysis}

Data were evaluated using the SPSS software program (version 12; SPSS Inc., Chicago, IL). Pearson's $\chi^{2}$ test and Fisher's exact test were used to detect differences in the prevalence of categorical variables and demographic, collaborative, and integrative variables between the physicians and practitioners. In addition, a Student's $t$ test was performed to determine whether any significant differences existed between the continuous variables between the 2 groups. $P$ values $<.05$ were deemed significant.

\section{Results}

Questionnaires were returned by 333 physicians and 241 CAM practitioners; response rates were $13 \%$ and $54 \%$, respectively. The respondents' characteristics are shown in Table 1.

After comparing both groups' attitudes toward collaborative teamwork in the areas of clinical practice, research, and education, we found that the CAM practitioners were significantly more supportive of collaborations between conventional and CAM health care providers than were the physicians in all 3 areas (Table 2).

Both groups had similar attitudes toward the potential integration of CAM into a primary care clinic and designated family physicians as the primary referral source in a hypothetical integrative medicine clinic. Nevertheless, the CAM practitioners were more supportive than the physicians of the family physician having a major role in CAM referrals ( $82 \%$ vs $63 \%$, respectively; $P<.0001)$. In both groups, more respondents indicated that CAM practitioners, not other health care providers, should offer CAM treatments in a hypothetical integrative medicine primary care setting (Table 3).

Table 4 shows notable differences in the attitudes of physicians and CAM practitioners toward 4 theoretical models of collaboration as described in the questionnaire. For example, physicians were significantly more supportive of teamwork that would be directed and coordinated by a physician than were the CAM practitioners (43\% vs $19 \%$, respectively; $P=.0001)$. In contrast, the CAM practitioners mostly supported codirected teamwork than were physicians $(21 \%$ vs $37 \%$, respectively; $P=.0001)$. However, as a secondary option, both groups supported a model in which the director of the treatment team was determined based on the distinctive characteristics of the patient's disease and condition.

According to our analyses, more than $60 \%$ of the respondents in both groups (219 of 332 physicians and 150 of 233 CAM practitioners) indicated 


\begin{tabular}{|c|c|c|}
\hline Characteristic & $\begin{array}{c}\text { Primary Care } \\
\text { Physicians }(\mathrm{n}=333)\end{array}$ & $\begin{array}{c}\text { CAM } \\
\text { Practitioners } \\
(\mathrm{n}=241)\end{array}$ \\
\hline \multicolumn{3}{|l|}{$\operatorname{Sex}^{*}(\mathrm{n}[\%])$} \\
\hline Male & $187(58)$ & $95(41)$ \\
\hline Female & $134(42)$ & $137(59)$ \\
\hline Age (mean $\pm \mathrm{SD}[$ median]) & $47.7 \pm 7.2(48)$ & $40.2 \pm 9.4(38)$ \\
\hline \multicolumn{3}{|l|}{ Medical specialty† (n [\%]) } \\
\hline Specialists & $265(80) \neq$ & \\
\hline Family medicine & $105(32)$ & \\
\hline Internal medicine & $47(14)$ & \\
\hline Pediatrics & $9(3)$ & \\
\hline \multicolumn{3}{|l|}{ CAM modality $(\mathrm{n}[\%]) \dagger$} \\
\hline Movement/manual healing & & $124(51) \$$ \\
\hline Traditional Chinese medicine & & $88(37)$ \\
\hline Naturopathy & & $29(12)$ \\
\hline Homeopathy & & $10(4)$ \\
\hline Herbal medicine & & $9(4)$ \\
\hline Chiropractic & & $8(3)$ \\
\hline Healing & & $4(2)$ \\
\hline Meditation & & $3(1)$ \\
\hline
\end{tabular}

Data analysis were performed by $t$ test. CAM, complementary and alternative medicine; SD, standard deviation.

*Three hundred twenty-one of 333 primary care physicians and 232 of 241 CAM practitioners reported this data.

†Respondents reporting any kind of medical or CAM specialty, which include one or more of the fields specified here.

$\ddagger$ Eighty-eight physicians $(27.3 \%)$ reported having studied CAM, with experience ranging from basic introductory courses to full programs. Twenty-four physicians $(7.7 \%)$ reported practicing CAM. Fifty-two percent reported having used CAM treatments over the past year.

\$Thirty-one (13\%) of the CAM practitioners were physicians.

that a referral or medical letter would be the best way for physicians and CAM practitioners to communicate about mutual patients. Although both groups preferred using a referral letter over other means of communication (such as telephone calls, e-mails, and direct meetings), more CAM practitioners than physicians supported these alternate methods. We used a 7-point scale to assess physicians' and practitioners' readiness to communicate with each other ("the other practitioner") when giving treatment to the same patient. We found that the CAM practitioners' self-ratings of readiness to communicate with "the other practitioner" were higher than the self-ratings of the physicians for this question (median, 5 out of 7 vs 4 out of 7 points, respectively; mean $\pm \mathrm{SD}, 4.55 \pm 1.62$ vs $4.21 \pm 1.86$, respectively; $P=.025$ ). Nevertheless, CAM practitioners reported they were less likely than the physicians to ask their patients about communicating with "the other practitioner" in reality (median, 2 out of 7 vs 3 out of 7 , respectively; mean $\pm \mathrm{SD}, 2.30 \pm 1.55$ vs $3.18 \pm 1.74$, respec- tively; $P<$.0001). Both groups estimated that their patients were interested in their health care providers engaging in dialogue (median, 4 out of 7 (for both groups); mean $\pm \mathrm{SD}, 4.10 \pm 1.48$ vs $4.38 \pm$ 1.56 , respectively; $P=.07$ ). In addition, both groups indicated they would be more ready to write a letter to "the other practitioner" if they had first received a referral letter than if they had not. In addition, physicians and CAM practitioners shared similar expectations regarding "the other practitioner" when administering treatment to the same patient: the principal expectation was that the other practitioner be willing to participate in consultations and construct a treatment plan together.

\section{Discussion}

In this study we surveyed the attitudes of primary care physicians and CAM practitioners toward the potential integration of CAM in a primary care setting. We based the perception of CAM integration into a primary care setting on the characteris- 
Table 2. Areas in Which Respondents Were Interested in Collaborative Teamwork Between Conventional and Complementary and Alternative Medicines

\begin{tabular}{lcrr}
\hline & \multicolumn{3}{c}{ Participants* (n [\%]) } \\
\cline { 2 - 3 } Area & $\begin{array}{c}\text { Primary Care } \\
\text { Physicians (n = 328) }\end{array}$ & $\begin{array}{c}\text { CAM Practitioners } \\
(\mathrm{n}=226)\end{array}$ & $P$ \\
\hline Clinical practice & $226(69)$ & $174(77)$ & .0430 \\
Scientific research & $50(15)$ & $95(42)$ & $<.0001$ \\
Medical education & $8(2)$ & $61(27)$ & $<.0001$ \\
\hline
\end{tabular}

Data analysis was performed by Pearson's $\chi^{2}$ test. CAM, complementary and alternative medicine.

*Three hundred twenty-eight of 333 primary care physicians and 226 of 241 CAM practitioners responded this question. Respondents were able to choose several options, thus the sum of percents exceeds $100 \%$.

tics that are common to both primary care and CAM, such as being patient-centered, being rooted in a holistic biopsychosocial agenda, and being characterized by a collaborative and integrative approach. We showed that both physicians and CAM providers envisioned the family physician as having an important role in CAM referrals if such treatment is made available in the clinic.

This study shows that primary care physicians and CAM practitioners share a positive view of communication between the "other practitioner" and themselves concerning mutual patients. Respondents in both groups highly supported the use of referral or medical letters and specifically stated that receiving a medical or referral letter from a patient's "other practitioner" would increase their readiness to respond in kind.

Sherman et $\mathrm{al}^{9}$ found similar results from a study of acupuncturists, who discussed approximately $50 \%$ of their physician-referred patients with the physicians, whereas they involved the patients' physician with only $12 \%$ of their other patients. Our report suggests that a referral letter from a physician to a CAM practitioner does more than communicate clinical information but that it may also affect the formation of a collaboration between physicians and CAM practitioners.

In our study, both groups reported a low rate of inter-profession communication in current daily practice, although they indicated that their patients might support it. More studies are needed to verify whether patients do support communication between their physicians and CAM practitioners. Such studies should also look at patient expectations regarding such dialogue as it relates to all 3 parties involved in treatment. ${ }^{19}$

Our study further showed that both groups expect collaboration, not communication only, regarding mutual patients. An example of multidisciplinary collaboration is the approach characterized

Table 3. Respondents' Attitudes to the Question, If Complementary and Alternative Medicine Was Provided in a Primary Care Clinic, Who Should Offer Complementary and Alternative Medicine Treatment?

\begin{tabular}{lccc}
\hline & \multicolumn{3}{c}{ Participants* $(\mathrm{n}[\%])$} \\
\cline { 2 - 3 } CAM Provider & $\begin{array}{c}\text { Primary Care } \\
\text { Physicians } \\
(\mathrm{n}=327 ; 327 \text { responses })\end{array}$ & $\begin{array}{c}\text { CAM Practitioners } \\
(\mathrm{n}=234 ; 264 \text { responses })\end{array}$ & $P$ \\
\hline CAM practitioner non-MD & $135(40.9)$ & $199(75.4)$ & $<.0001$ \\
CAM practitioner MD & $119(36.1)$ & $31(11.7)$ & $<.0001$ \\
Family physician in the clinic & $48(14.5)$ & $9(3.4)$ & NS \\
Nurse & $6(1.8)$ & $5(2.1)$ & NS \\
Pharmacist & $4(1.2)$ & $16(1.9)$ & NS \\
Other & $15(4.5)$ & $16(6.1)$ & \\
\hline
\end{tabular}

Data analysis was performed by Pearson's $\chi^{2}$ test. NS, not significant; CAM, complementary and alternative medicine.

*Three hundred twenty-seven of 333 primary care physicians and 234 of 241 CAM practitioners responded this question. Respondents were asked to focus on merely one option but were able to choose several options. Thus, number of responses is higher than the number of respondents. The data in the table refer to number of responses. 
Table 4. Respondents' Attitudes to the Question, How Do You Perceive Conjoint Physician- Complementary and Alternative Medicine Practitioner Teamwork?

\begin{tabular}{|c|c|c|c|}
\hline \multirow[b]{2}{*}{ Team Director } & \multicolumn{2}{|c|}{ Participants* (n [\%]) } & \multirow[b]{2}{*}{$P$} \\
\hline & $\begin{array}{c}\text { Primary Care Physicians } \\
(\mathrm{n}=283 ; \text { responses }=298)\end{array}$ & $\begin{array}{c}\text { CAM Practitioners } \\
(\mathrm{n}=198 ; \text { responses }=227)\end{array}$ & \\
\hline Physician $†$ & $127(42.6)$ & $43(18.9)$ & .0007 \\
\hline CAM practitioner $†$ & $8(2.7)$ & $12(5.3)$ & NS \\
\hline Codirected $\neq$ & $62(20.8)$ & $83(36.6)$ & .0001 \\
\hline Directed by either physician or CAM practitioner $\$$ & $86(28.9)$ & $60(26.4)$ & NS \\
\hline
\end{tabular}

Data analysis was performed by Pearson's $\chi^{2}$ test. NS, not significant; CAM, complementary and alternative medicine.

*Two hundred eighty-three of 333 primary care physicians and 198 of 241 CAM practitioners responded this question. Respondents were asked to focus on merely one option but were able to choose several options. Thus, number of responses is higher than the number of respondents. The data in the table refer to number of responses.

†The head of the team is a physician (or CAM practitioner) that directs and coordinates the treatment.

†The physician and CAM practitioner have equal standing, with neither of them heading the team.

$\$$ The head of the team is determined by the unique characteristics of the patient and his/her illness.

by the 5 NHS general practices in the United Kingdom, which integrate anthroposophic and conventional medicine. ${ }^{20}$ However, based on our results that indicated physicians favored a physician-directed model whereas practitioners favored a codirected model, we believe that physicians and CAM practitioners perceive collaboration differently. This discrepancy is not remarkable because teams composed of professionals from different fields may experience conflicts relating to status, power, and different understandings of the concepts and nomenclature used in different modalities. ${ }^{21}$ Caspi et $\mathrm{al}^{22}$ proposed the Tower of Babel metaphor as a way of characterizing the "language" gap between conventional medicine and CAM schools of thought. This communication gap may also be overcome through the implementation of educational initiatives for both physicians and CAM practitioners. We suggest that such efforts focus not only on improving knowledge and attitudes but also on obtaining the skills necessary to write a medical letter as a channel for mutual communication.

Our study had significant limitations that may have influenced our findings. For example, the physician group may have been affected by a selection bias resulting from the low response rate and high prevalence of personal CAM use among the responding physicians over the previous year. Low interest in or opposition to CAM may have been the reason for other physicians' not responding. The low response rate may also have been a result of the physicians' unfamiliarity with a new Webbased questionnaire collection system. Physicians' familiarity with CAM in this study (27\%) is comparable with the findings in another study in Israel ( $25 \%$ of 165 physicians), ${ }^{23}$ so the low response rate is not necessarily a source of bias. In any case, the results of the present study may reflect the attitudes of a physician subpopulation that favors CAM more than does their group as a whole.

Therefore, we recommend interpreting the study results with caution; our study participants may be more receptive to and experienced in integrative medicine than their colleagues. Moreover, our study results may reflect the attitudes of a minority of physicians (13\% response rate) who have more interest in issues relating to communication with CAM providers.

It may be instructive to investigate whether the physicians who did not respond to the questionnaire are less familiar with CAM providers or less convinced than CAM practitioners of the importance inter-professional collaboration. Studies in larger populations of physicians and CAM practitioners, including physicians unfamiliar with CAM, may clarify the importance of the communication between the 2 groups and the influence of this collaboration on physician-patient communication.

In summary, this study suggests that primary care physicians and CAM practitioners are willing to collaborate and communicate with each other, especially concerning a mutual patient. Writing a referral or medical letter is their preferred way to communicate. Educational programs for physicians and CAM practitioners should focus on aspects of communication and practical methods in writing a referral or medical letter in the scope of CAM. 
We thank Anat Klein, ND, and Dr. Chen Shapira for their generous support and encouragement; Ronit Leiba for statistical analysis; Dr. Adva Lear, medical director of Herman Clinic, and associated clinical staff members for their support and collaboration with the team of researchers; the research team from the International Center and College of Natural Complementary Medicine, including Dalia Vardi, Danit Steinberg, Ira Videtski, Uri Meir Chizik, Einav Damari, Moran Barkan, Hagit Confarti, Yoram Sendak, Riki Caspi, Orit Zarfati, and Pnina Sneitzer; Hamichlala Leminhal for financial support of the statistical work-up; and Yael Bruno, Marianne Steinmetz, and Don Norwood for editing the manuscript.

\section{References}

1. Bell IR, Caspi O, Schwartz GE, et al. Integrative medicine and systemic outcomes research: issues in the emergence of a new model for primary health care. Arch Intern Med 2002;162:133-40.

2. Richardson MA, Masse LC, Nanny K, Sanders C. Discrepant views of oncologists and cancer patients on complementary/alternative medicine. Support Care Cancer 2004;12:797-804.

3. Pappas S, Perlman A. Complementary and alternative medicine. The importance of doctor-patient communication. Med Clin North Am 2002;86:1-10.

4. Rees L, Weil A. Integrated medicine. BMJ 2001;322: 119-20.

5. Rosenthal DS, Dean-Clower E. Integrative medicine in hematology/oncology: benefits, ethical considerations, and controversies. Hematology Am Soc Hematol Educ Program 2005:;491-7.

6. Robinson A, McGrail MR. Disclosure of CAM use to medical practitioners: a review of qualitative and quantitative studies. Complement Ther Med 2004; 12:90-8.

7. Adler SR, Fosket JR. Disclosing complementary and alternative medicine use in the medical encounter: a qualitative study in women with breast cancer. J Fam Pract 1999;48:453-8.

8. Crock RD, Jarjoura D, Polen A, Rutecki GW. Confronting the communication gap between conventional and alternative medicine: a survey of physicians' attitudes. Altern Ther Health Med 1999;5: 61-6.

9. Sherman KJ, Cherkin DC, Eisenberg DM, Erro J, Hrbek A, Deyo RA. The practice of acupuncture: who are the providers and what do they do? Ann Fam Med 2005;3:151-8.

10. Greene BR, Smith M, Allareddy V, Haas M. Referral patterns and attitudes of primary care physicians towards chiropractors. BMC Complement Altern Med 2006;6:5.

11. Lewith GT, Hyland M, Gray SF. Attitudes to and use of complementary medicine among physicians in the United Kingdom. Complement Ther Med 2001; 9:167-72.

12. National Center for Complementary and Alternative Medicine. Expanding horizons of healthcare: Fiveyear strategic plan, 2001-2005. Available at http:// nccam.nih.gov/about/plans/fiveyear/index.htm. Accessed 25 April 2007.

13. Vohra S, Feldman K, Johnston B, Waters K, Boon $\mathrm{H}$. Integrating complementary and alternative medicine into academic medical centers: experience and perceptions of nine leading centers in North America. BMC Health Serv Res 2005;5:78.

14. Kmietowicz Z. Complementary medicine should be integrated into the NHS. BMJ 1997;315:1111-6.

15. Smallwood C. The role of complementary and alternative medicine in the NHS. An investigation into the potential contribution of mainstream complementary therapies to healthcare in the UK (2005). Available at http://www.freshminds.co.uk/PDF/ THE\%20REPORT.pdf. Accessed 5 June 2007.

16. Committee on the Use of Complementary and alternative Medicine by the American Public. Complementary and alternative medicine (CAM) in the United States. Institute of Medicine of the National Academies: Washington, DC; 2005.

17. Clalit Health Services: overview. Available at http:// www.microsoft.com/israel/casestudies/clalit_sql. mspx. Accessed 5 June 2007.

18. BDI-COFACE report on CAM treatments in Israel, 2005. Available at http://www.ynet.co.il/articles/ 0,7340, L-3296668,00.html. Accessed 25 April 2007.

19. Adler SR. Relationships among older patients, CAM practitioners, and physicians: the advantages of qualitative inquiry. Altern Ther Health Med 2003;9:104-10.

20. Ritchie J, Wilkinson J, Gantley M, Feder G, Carter Y, Formby J. A model of integrated primary care: anthroposophic medicine. London: National Centre for Social Research; 2001.

21. Robinson M, Cottrell D. Health professionals in multi-disciplinary and multi-agency teams: changing professional practice. J Interprof Care 2005;19:54760.

22. Caspi O, Bell IR, Rychener D, Gaudet TW, Weil AT. The Tower of Babel: communication and medicine: an essay on medical education and complementary-alternative medicine. Arch Intern Med 2000;160:3193-5.

23. Giveon SM, Liberman N, Klang S, Kahan E. A survey of primary care physicians' perceptions of their patients' use of complementary medicine. Complement Ther Med 2003;11:254-60. 